\title{
Démographie et performances zootechniques des élevages bovins traditionnels au Nord Bénin
}

\author{
Roukayath Chabi Toko ${ }^{1 *}$ Anselme Adégbidi ${ }^{2}$ \\ Philippe Lebailly ${ }^{1}$
}

\section{Mots-clés}

Bovin Borgou, troupeau, performance de reproduction, production de bétail, Bénin

Accepted : 11 January 2016 Published : 4 July 2016

\section{Résumé}

L'étude a été menée auprès de 150 éleveurs peuls dans les départements du Borgou et de l'Alibori au Bénin. Elle a eu pour objectif de déterminer les paramètres zootechniques des élevages traditionnels de bovins. L'enquête a permis de recueillir les informations sur les caractéristiques des troupeaux bovins. Les résultats on montré une forte proportion de femelles (76\%), une taille moyenne des troupeaux (66 animaux) et la prévalence de la race Borgou (86\%). Les troupeaux ont par ailleurs présenté de faibles performances d'élevage, caractérisées par un taux important de mortalité des veaux $(10 \%)$, et de faibles paramètres de reproduction, avec des taux de mise bas et de fécondité de $64 \%$. Au cours d'une année, 0,57 veau est en moyenne sevré par sa mère. Les faibles performances des élevages bovins dans les pays côtiers comme le Bénin concordent avec celles des régions pastorales qui sont complémentaires en termes d'approvisionnement des marchés ouest africains en viande.

- Pour citer cet article : Chabi Toko R., Adégbidi A., Lebailly P., 2016. Demography and zootechnical performance of traditional cattle farming in Northern Benin [in French]. Rev. Elev. Med. Vet. Pays Trop., 69 (1) : 33-39

\section{INTRODUCTION}

L'économie du Bénin, à l'instar des pays d'Afrique de l'Ouest, est basée en majorité sur la production agricole. L'agriculture a contribué à $32,7 \%$ au produit intérieur brut (PIB) en 2012. Le sous-secteur élevage a rapporté la même année 180,6 millions de dollars US, soit 2,4 \% du PIB (FAO, 2015). L'élevage est pratiqué par la majorité des populations agricoles. Il constitue la seconde activité économique après les cultures (De Haan et al., 1997). Le cheptel national est estimé à 4,6 millions de têtes dont 2,1 millions de bovins et 2,5 millions de petits ruminants en 2012. Il se concentre à $85 \%$ au nord du pays avec $63 \%$ dans les départements du Borgou et de l'Alibori

\footnotetext{
1. Université de Liège, Gembloux Agro-Bio Tech, Unité d'économie et de développement rural, passage des Déportés, 2 B-5030, Gembloux, Belgique.

2. Université d'Abomey-Calavi, Faculté des sciences agronomiques, Département d'économie, de socio-anthropologie et de communication, Cotonou, Bénin.

* Auteur pour la correspondance

Tél. : +22995840204 ou +32465867780

Email : rchabitoko@doct.ulg.ac.be
}

(FAO, 2013). L'exploitation des bovins et des petits ruminants y est commune et repose sur les pâturages naturels et les résidus de récolte (Djenontin et al., 2002 ; Djenontin, 2010). Les Peuls possèdent $95 \%$ du cheptel bovin national et sont ainsi les acteurs incontournables pour la fourniture en protéines animales du pays (Dehoux et Hounsou-Vè, 1993). Les bovins sont les principaux pourvoyeurs de viande et de lait des populations locales. Diverses études ont été réalisées pour caractériser les performances des bovins élevés en station dans des conditions semi-intensives (Alkoiret et al., 2010b ; Gbangboché et Alkoiret, 2011). Les différents paramètres collectés ne représentent qu'en partie la réalité des troupeaux bovins béninois. Les données existantes sur les troupeaux bovins traditionnels ne concernent que la commune de Gogounou (Alkoiret et al., 2009 ; Alkoiret et al., 2010a) ou ne sont pas actualisées (Dehoux et Hounsou-Vè, 1993). Cette étude vise ainsi à fournir des données récentes sur des troupeaux bovins traditionnels (élevés de manière extensive sur parcours naturels) dans la partie du pays où ils sont concentrés pour leur prise en compte dans des actions concernant le développement de l'élevage bovin au Bénin. Elle a eu pour objectif de déterminer les paramètres zootechniques de l'élevage de bovins traditionnel au nord-est du Bénin, et plus précisément de déterminer les paramètres démographiques, la composition des races et les performances d'élevage. 


\section{MATERIEL ET METHODES}

\section{Milieu d'étude}

La recherche a été menée dans les départements du Borgou et de l'Alibori situés entre $02^{\circ} 04^{\prime}$ et $03^{\circ} 33^{\prime} \mathrm{E}$, et $09^{\circ} 24^{\prime}$ et $12^{\circ} 08^{\prime} \mathrm{N}$. Le climat dans la zone est de type sahélo-soudanien et soudano-guinéen, avec deux saisons, une saison sèche et une saison des pluies. On y distingue trois zones agroécologiques (De Haan et al., 1997). La première est caractérisée par un climat soudano-sahélien. La saison des pluies dure de mai à octobre avec des précipitations annuelles variant de 700 à $900 \mathrm{~mm}$. La végétation est arborée, arbustive et herbeuse avec une prédominance d'épineux. La deuxième a un climat soudanien. La saison des pluies s'étend de mai à octobre avec une pluviométrie annuelle comprise entre 800 et $1200 \mathrm{~mm}$. C'est une zone de savane arborée, arbustive et herbeuse. La troisième est caractérisée par un climat soudanien. La saison des pluies s'étend d'avril à novembre avec une pluviométrie annuelle oscillant entre 900 et $1300 \mathrm{~mm}$. Dans cette zone, la végétation est celle d'une forêt secondaire clairsemée de type savane soudano-guinéenne. Ces deux départements concentrent $55 \%$ de Peuls (Insae, 2003). Les Peuls forment un important groupe socioculturel $(81,4$ à $96,2 \%)$ parmi les populations qui pratiquent l'élevage du bétail en Afrique de l'Ouest (Dehoux et Hounsou-Vè, 1993 ; Alkoiret et al., 2009 ; IIED / SOS Sahel, 2010).

Trois communes, appartenant chacune à une zone agroécologique, ont été sélectionnées en fonction de l'importance de leur effectif bovin (FAO, 2015) et de l'importance de la communauté peule (Insae, 2003) afin de tenir compte de la diversité de la région. Il s'agissait de Kalalé (dans la troisième zone) dans le Borgou, et de Banikoara (dans la deuxième zone) et de Malanville (dans la première zone) dans l'Alibori (figure 1). Pour chaque commune, les trois premiers arrondissements du point de vue de l'effectif du cheptel bovin ont été retenus. Un village a été choisi par arrondissement de manière à avoir un nombre suffisant d'éleveurs de bovins peuls au sein dudit village.

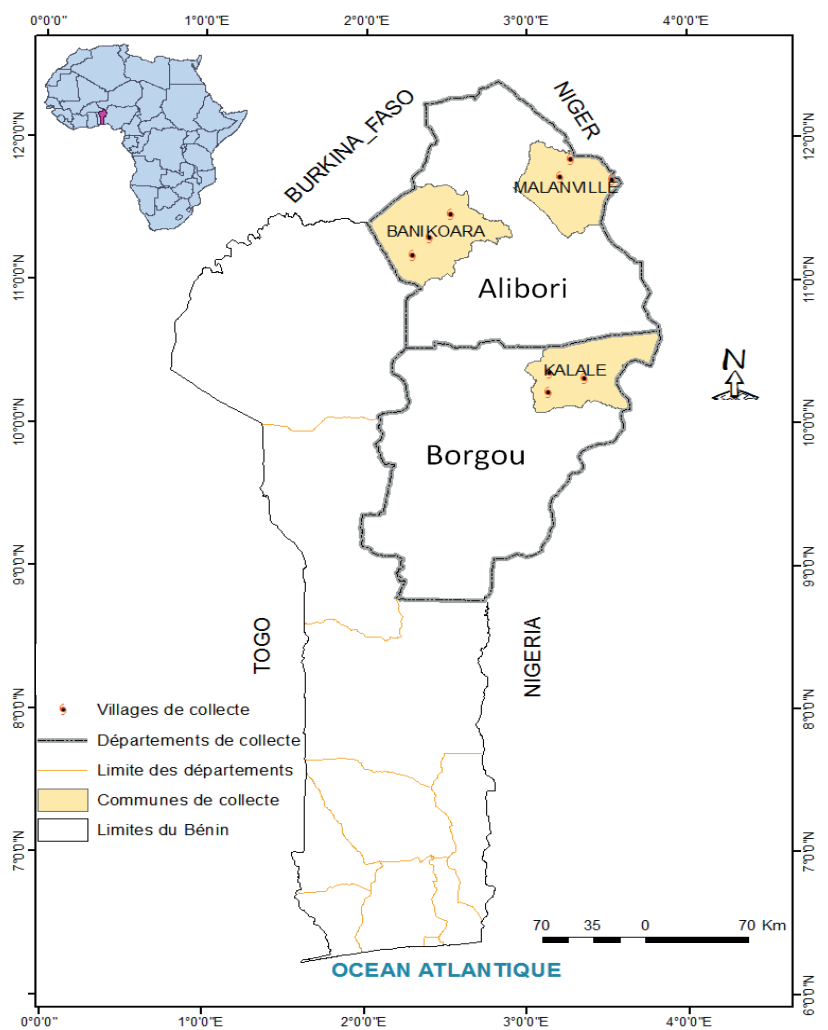

Figure 1 : situation de la zone d'étude au Bénin et en Afrique de l'Ouest (source : d'après le Fond topograhique du Bénin, 1992).

\section{Echantillonnage et méthode d'enquête}

Les troupeaux bovins d'un échantillon de 150 éleveurs peuls, soit 50 éleveurs par commune, ont été enquêtés du 3 au 26 juin 2013. Dans les villages où l'enquête s'est déroulée, le nombre d'éleveurs sélectionnés représentait environ $50 \%$ de l'effectif des éleveurs de bovins peuls du village. Au sein des trois villages ciblés par commune, les troupeaux de bovins appartenant aux éleveurs peuls ont été choisis de façon aléatoire.

Les informations sur les troupeaux ont été recueillies sur les systèmes de production (Ferraton et Touzard, 2009). L'enquête transversale rétrospective basée sur la mémoire des éleveurs a été utilisée et la « méthode des douze derniers mois » (Lesnoff, 2013) a permis de dégager les caractéristiques démographiques des cheptels (taille et structure des troupeaux, reproduction, mortalité).

Les performances annuelles des troupeaux, comme les paramètres de reproduction, le taux de mortalité des jeunes et la productivité numérique au sevrage, ont été calculées d'après les formules de Ferraton et Touzard (2009) :

taux de mise bas $=$ nombre de femelles mettant bas $\mathrm{x} 100 / \mathrm{nb}$. de femelles mises à la reproduction

taux de prolificité $=$ nombre de petits nés $\mathrm{x} 100 / \mathrm{nb}$. de femelles mettant bas

taux de fécondité $=$ nombre de petits nés $\mathrm{x} 100 / \mathrm{nb}$. de femelles mises à la reproduction

ou taux de fécondité = taux de mise bas $\mathrm{x}$ taux de prolificité

taux de mortalité des jeunes $=$ nombre de petits morts $x 100 /$ nb. de petits nés

productivité numérique au sevrage = taux de fécondité $\mathrm{x}(100$ - taux de mortalité des jeunes).

Pour obtenir les paramètres annuels, les ratios obtenus plus haut ont été divisés par l'intervalle entre deux mises bas mesuré en années. Cet intervalle entre mises bas est le plus souvent supérieur à un an dans les élevages traditionnels.

\section{Analyses statistiques}

Les données saisies dans le logiciel Excel ont été analysées avec le logiciel SPSS 20. La statistique descriptive (moyenne, écart type, fréquence) a permis de décrire les caractéristiques des troupeaux bovins. Afin de déterminer dans quelle mesure les paramètres zootechniques des élevages bovins étaient spécifiques à chaque zone, les tests du Chi carré et Anova ont été utilisés pour comparer les données des communes. Les variables quantitatives (effectif des troupeaux, effectif par sexe et par âge, âge de reproduction et différents taux de reproduction et de mortalité) ont été soumis à l'analyse de variances à un facteur et les communes où les enquêtes ont été réalisées a été l'unique source de variation. Les moyennes ont été comparées deux à deux grâce au test $t$ de Student. Les fréquences des variables nominales des communes ont été comparées avec le test du Chi carré.

\section{RESULTATS}

\section{Paramètres démographiques}

L'effectif des bovins par ménage enquêté a varié entre 20 et 748 animaux. La valeur médiane a été de 48 animaux et le mode de 30 animaux. L'effectif moyen de 66 animaux a présenté une grande variabilité (tableau I). La distribution en effectifs bovins montre que la majorité $(75 \%)$ des éleveurs possédait des troupeaux dont la taille maximale était de 79 animaux. Les effectifs en bovins ont présenté des valeurs significativement différentes entre les communes (tableau I). 
La structure par sexe des troupeaux bovins a montré une très forte proportion de femelles ( $76 \%$ ) dont $47 \%$ de vaches (tableau I). La proportion de veaux, de génisses et de vaches a été similaire dans les troupeaux des trois communes. La proportion de taurillons, de taureaux et de velles a été significativement différente entre les communes. Le pourcentage de taurillons dans les troupeaux de Kalalé et de Malanville a été significativement plus élevé que dans ceux de Banikoara. Quant aux taureaux, leur proportion (9\%) plus élevée dans les troupeaux de Kalalé a été significativement différente de celle enregistrée dans les troupeaux de Banikoara (6\%) et de Malanville (4\%). Les troupeaux de Banikoara ont présenté des proportions de velles significativement plus faibles.

\section{Composition en races des troupeaux bovins}

Quatre races ont été rencontrées dans les troupeaux bovins : le Borgou, le M'bororo, le Gudali et des métis Somba x Borgou (tableau II).

Le Borgou est issu du croisement stabilisé entre les taurins à cornes courtes d'Afrique occidentale (Somba ou Lagunaire) et le zébu White Fulani (Domingo, 1976). La robe est blanche ou mouchetée, généralement pie noir, parfois noire. Les éleveurs préfèrent cette race en raison de sa bonne production laitière, d'un bon rendement en carcasse et de sa prolificité.

Le Gudali est un zébu d'Afrique orientale et centrale, semblable au zébu à cornes courtes d'Afrique de l'Est du point de vue de la conformation, de l'origine et de la taille. Cet animal est réputé non seulement pour sa viande et sa qualité laitière, mais aussi pour sa résistance à de dures conditions environnementales. Il est caractérisé par de larges et grandes oreilles, l'absence de cornes ou quelques fois la présence de cornes de petite taille et d'un fanon pendant.

Le bovin Somba appartient aux taurins trypanotolérants à cornes courtes d'Afrique de l'Ouest. Son berceau est situé entre le nord-ouest du Bénin et le nord-est du Togo. Métissé avec le Borgou, il est élevé en raison de sa rusticité et de son caractère docile qui en font un bon animal pour le dressage (culture attelée).

Le M'bororo est un zébu d'Afrique de l'Ouest à cornes longues. Il peuple tout le Niger jusqu'au Tchad et le nord du Nigeria. C'est un animal de taille moyenne à grande, nerveux et d'une grande finesse. C'est un marcheur de grande transhumance. Les cornes, très longues, en forme de lyre, sont implantées haut avec la pointe dirigée vers l'arrière. La robe est uniforme, acajou foncé, les muqueuses sont claires (Meyer, 2013).

Au sein des troupeaux de bovins, $86 \%$ étaient composés de Borgou avec des proportions significativement plus faibles pour la commune de Banikoara (tableau II). Les troupeaux comportant plusieurs races étaient rencontrés fréquemment dans les deux communes de l'Alibori (Banikoara et Malanville). Les troupeaux exclusivement composés de métis Somba x Borgou étaient uniquement présents à Banikoara. La proportion de ces troupeaux y était significativement plus élevée que dans les deux autres communes. Les M'bororo et Gudali étaient présents au sein des cheptels de Malanville qui a des frontières avec le Niger et le Nigeria.

\section{Tableau}

Structure des troupeaux bovins dans les communes de Kalalé, Banikoara et Malanville au Bénin

\begin{tabular}{|c|c|c|c|c|c|c|}
\hline \multirow[t]{2}{*}{ 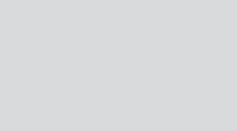 } & Age & Kalalé & Banikoara & Malanville & \multirow{2}{*}{$\begin{array}{l}\text { Total } \\
(\%)\end{array}$} & \multirow[t]{2}{*}{ Significativité } \\
\hline & & \multicolumn{3}{|c|}{ Moyenne \pm écart type $(\%)$} & & \\
\hline Effectif troupeau & & $58 \pm 66^{a}$ & $46 \pm 25^{a}$ & $93 \pm 102^{b}$ & $66 \pm 74$ & $* *$ \\
\hline Veau & $<1$ an & $11 \pm 5^{a}$ & $10 \pm 5^{a}$ & $10 \pm 4^{\mathrm{a}}$ & $11 \pm 5$ & NS \\
\hline Taurillon & 1 à 3 ans & $9 \pm 7^{a}$ & $5 \pm 4^{b}$ & $8 \pm 4^{a}$ & $7 \pm 5$ & $* * *$ \\
\hline Taureau & $>3$ ans & $8 \pm 5^{a}$ & $6 \pm 4^{b}$ & $4 \pm 1^{c}$ & $6 \pm 4$ & $* * *$ \\
\hline Velle & $<1$ an & $11 \pm 5^{a}$ & $16 \pm 6^{b}$ & $16 \pm 5^{b}$ & $14 \pm 6$ & $* * *$ \\
\hline Génisse & 1 à 3 ans & $14 \pm 6^{a}$ & $16 \pm 7^{a}$ & $15 \pm 6^{a}$ & $15 \pm 6$ & NS \\
\hline Vache & $>3$ ans & $46 \pm 12^{a}$ & $46 \pm 11^{a}$ & $47 \pm 7^{a}$ & $47 \pm 10$ & NS \\
\hline
\end{tabular}

Les effectifs des catégories d'animaux sont en pourcentage de l'effectif du troupeau.

Les valeurs sur une même ligne suivies de lettres différentes sont significativement différentes $(\mathrm{p}<0,05)$; ** : fortement significatif $(\mathrm{p}<0,01)$; *** : très fortement significatif $(\mathrm{p}<0,001)$; NS : non significatif $(\mathrm{p} \geq 0,05)$.

\section{Tableau II}

Races rencontrées dans les troupeaux bovins de Kalalé, Banikoara et Malanville au Bénin

\begin{tabular}{|c|c|c|c|c|c|}
\hline Race & $\begin{array}{c}\text { Kalalé } \\
(\%)\end{array}$ & $\begin{array}{c}\text { Banikoara } \\
(\%)\end{array}$ & $\begin{array}{c}\text { Malanville } \\
(\%)\end{array}$ & $\begin{array}{l}\text { Total } \\
(\%)\end{array}$ & Significativité \\
\hline Borgou & $98^{a}$ & $72 b$ & $88^{a}$ & 86 & $* *$ \\
\hline Métisse Somba x Borgou & $0^{\mathrm{a}}$ & $18^{b}$ & $0^{a}$ & 6 & $* * *$ \\
\hline Borgou et Gudali & $2^{a, b}$ & $0^{b}$ & $8^{a}$ & 3,3 & $*$ \\
\hline Borgou et métisse Somba x Borgou & $0^{a}$ & $8^{b}$ & $0^{\text {a }}$ & 2,7 & $*$ \\
\hline Borgou et $\mathrm{M}^{\prime}$ bororo & $0^{\mathrm{a}}$ & $0^{\mathrm{a}}$ & $2^{a}$ & 0,7 & NS \\
\hline Borgou, Gudali et M'bororo & $0^{\mathrm{a}}$ & $0^{\text {a }}$ & $2^{a}$ & 0,7 & NS \\
\hline
\end{tabular}

Les valeurs sur une même ligne suivies de lettres différentes sont significativement différentes $(\mathrm{p}<0,05) ; *$ : significatif $(\mathrm{p}<0,05) ; * *$ : fortement significatif $(\mathrm{p}<0,01)$; $* * *$ : très fortement significatif $(p<0,001) ; \mathrm{NS}$ : non significatif $(\mathrm{p} \geq 0,05)$. 


\section{Performances de reproduction}

Chez les mâles comme chez les femelles, l'âge à la première mise à la reproduction était de quatre ans dans les trois communes. Les femelles restaient toutefois beaucoup plus longtemps que les mâles dans le troupeau (tableau III). Les performances de reproduction des troupeaux étaient généralement faibles avec un taux de mise bas de $64 \%$, de prolificité de $83 \%$ et de fécondité de $64 \%$. A Banikoara les taux de mise bas $(71 \%)$ et de fécondité $(72 \%)$ ont été plus élevés que ceux obtenus à Kalalé et à Malanville. Le taux de mortalité des veaux pour l'ensemble de la zone d'étude a été de $10 \%$. Il existait cependant des disparités entre les communes. Le taux enregistré à Kalalé (18\%) a présenté des valeurs significativement plus élevées que celles relevées à Banikoara (4\%) et à Malanville (8\%). En moyenne, 0,6 veau a été sevré par an par sa mère dans les troupeaux bovins traditionnels ; les troupeaux de Banikoara ont présenté la valeur moyenne la plus élevée $(0,7)$; cette valeur a été significativement différente de celles enregistrées à Malanville et à Kalalé (respectivement 0,6 et 0,5). Dans l'ensemble, les troupeaux bovins de Banikoara ont présenté les meilleures performances d'élevage de la zone d'étude.

\section{DISCUSSION}

Les différentes stratégies d'adaptation des éleveurs à leurs conditions agroécologiques pourraient expliquer les différences significatives d'effectifs bovins au niveau des trois communes. En effet, les éleveurs situés dans la commune la plus aride caractérisée par un climat soudano-sahélien (Malanville) ont significativement plus d'animaux pour permettre à leurs troupeaux de supporter les chocs dus aux sécheresses qui sont plus récurrentes dans la zone. Cet effectif élevé serait aussi dû à l'existence de peu d'espaces propices à l'agriculture, les éleveurs disposant ainsi davantage d'espaces pastoraux. De plus, les troupeaux dans cette commune doivent parcourir des distances plus longues que ceux des deux autres communes lors de la transhumance. Ceci augmente alors la probabilité d'enregistrer plus de pertes d'animaux. En outre, du fait de l'éloignement de leur campement d'attache, ceux qui vont en transhumance à partir de cette commune dépendent davantage des produits issus de l'élevage bovin (animaux sur pied et lait) pour leur survie ; de grands effectifs permettent d'assurer un certain niveau de vie tout en préservant le troupeau. C'est une stratégie de résilience que les éleveurs des deux autres communes n'ont pas adoptée car ils se trouvent dans des zones au climat plus favorable, mais aussi parce qu'il y a concurrence entre les espaces pastoraux et agricoles. Le type de système d'élevage pratiqué à Malanville qui tend beaucoup plus vers le pastoralisme pourrait également expliquer l'existence de troupeaux de grande taille.

La structure des troupeaux bovins traduit l'objectif premier de l'éleveur qui est de vivre de façon durable de son troupeau. Pour cela, il lui faut assurer la pérennité de son cheptel avec un effectif élevé en femelles reproductrices. Ces dernières assurent le renouvellement du troupeau aussi bien en mâles, utilisés pour la traction animale, qu'en femelles assurant le remplacement des femelles en fin de carrière. Le petit nombre de mâles dans le troupeau de bovins est la conséquence de la vente de ceux-ci pour régler les problèmes d'argent mais également pour faciliter la gestion du troupeau. En effet, les premiers animaux que les Peuls vendent sont, la plupart du temps, les taurillons, suivis par les vaches réformées. Dehoux et Hounsou-Vè (1993) rapportent que les éleveurs vendent très tôt les taurillons qui sont utilisés comme animaux de trait. Ces éleveurs n'ont souvent pas recours à la castration car le Borgou est une race placide. De plus, les agriculteurs préfèrent utiliser les animaux entiers pour la culture attelée (Dehoux et Hounsou-Vè, 1993).

Par ailleurs, cette structure met aussi en exergue la fonction de production mixte des bovins Borgou. Le lait procure des revenus réguliers à la femme et au ménage, et la vente des jeunes mâles permet à l'homme de subvenir à ses besoins mais aussi à ceux de la famille. L'effectif des taurillons dans les troupeaux de Banikoara pourrait être dû à la forte demande pour la culture attelée dans cette commune. En effet, Banikoara est la première commune productrice de coton au Bénin en superficie mais également en terme de production. Mis à part le labour avec un tracteur, la culture attelée est couramment utilisée pour le sarclo-buttage et le labour. Les bœufs de trait servent également dans le transport du coton-graine à un endroit précis du champ car de grandes superficies sont emblavées (entre 2,5 et $50 \mathrm{ha}$ ).

Des structures similaires ont été rapportées par d'autres auteurs pour les troupeaux bovins du nord du Bénin. Ces troupeaux sont constitués entre 65 et $75 \%$ de femelles (Dehoux et Hounsou-Vè, 1993 ; Alkoiret et al., 2010b). De même, dans la sous-région ouest africaine les vaches dominent les troupeaux (45 à $80 \%$ ) et sont généralement utilisées pour la production du lait dans les cheptels bovins du Sénégal (Ejlertsen et al., 2011), les troupeaux bovins des petits producteurs laitiers de Gambie (Somda et al., 2005) et les troupeaux bovins des Peuls agropasteurs du Nigeria (Daodu et al., 2009 ; Akpa et al., 2012).

\section{Tableau III}

Paramètres de reproduction des troupeaux bovins de Kalalé, Banikoara et Malanville au Bénin

\begin{tabular}{|c|c|c|c|c|c|}
\hline Paramètre & Kalalé & Banikoara & Malanville & \multirow[t]{2}{*}{ Total } & \multirow[t]{2}{*}{ Significativité } \\
\hline \multicolumn{4}{|c|}{ Moyenne \pm écart type } & & \\
\hline Age de reproduction des mâles (ans) & $4 \pm 1^{\mathrm{a}}$ & $3,6 \pm 1^{b}$ & $3 \pm 0^{c}$ & $4 \pm 1$ & $* * *$ \\
\hline Age de reproduction des femelles (ans) & $4 \pm 1^{\mathrm{a}}$ & $3,6 \pm 1^{b}$ & $2,9 \pm 0^{c}$ & $4 \pm 1$ & $* * *$ \\
\hline Age de réforme des mâles (ans) & $12 \pm 3^{a}$ & $10 \pm 1^{b}$ & $9,9 \pm 1^{b}$ & $10 \pm 2$ & $* * *$ \\
\hline Age de réforme des femelles (ans) & $15 \pm 3^{\mathrm{a}}$ & $13 \pm 3^{b}$ & $12,7 \pm 1^{b}$ & $14 \pm 3$ & $* * *$ \\
\hline Taux de mise bas (\%) & $60 \pm 16^{a}$ & $71 \pm 14^{b}$ & $60 \pm 10^{\mathrm{a}}$ & $64 \pm 15$ & $* * *$ \\
\hline Taux de prolificité (\%) & $86 \pm 15^{a}$ & $90 \pm 13^{a}$ & $74 \pm 11^{b}$ & $83 \pm 15$ & $* * *$ \\
\hline Taux de fécondité (\%) & $60 \pm 16^{a}$ & $72 \pm 14^{b}$ & $60 \pm 10^{\mathrm{a}}$ & $64 \pm 15$ & $* * *$ \\
\hline Taux de mortalité (\%) & $18 \pm 17^{a}$ & $4 \pm 8^{b}$ & $8 \pm 7^{b}$ & $10 \pm 13$ & $* * *$ \\
\hline PNS (\%) & $48 \pm 20^{a}$ & $68 \pm 16^{b}$ & $53 \pm 11^{\mathrm{a}}$ & $57 \pm 18$ & $* * *$ \\
\hline
\end{tabular}

Les valeurs sur une même ligne suivies de lettres différentes sont significativement différentes $(\mathrm{p}<0,05) ; * * *$ : très fortement significatif $(\mathrm{p}<0,001)$.

PNS : productivité numérique au sevrage 
Les troupeaux bovins du nord du Bénin ainsi que ceux de la sousrégion ouest africaine ont des structures similaires caractérisées par la dominance des femelles. Cette structure n'a pratiquement pas connu d'évolution, comme le montrent la synthèse d'Otte et Chilonda (2002) et le tableau IV sur les troupeaux de bovins d'Afrique subsaharienne au cours du temps ; les éleveurs du nord du Bénin et ceux des pays sahéliens poursuivent le même objectif, c'est-à-dire la pérennisation des troupeaux bovins. Cet objectif commun permet de répondre à la demande sans cesse croissante en viande des pays côtiers, où la pression des glossines et la pression démographique sont élevées. Les troupeaux bovins du nord du Bénin permettent par leur structure d'alimenter le marché de viande des régions du sud du pays avant que les troupeaux transhumants des pays sahéliens n'arrivent. Une partie des taurillons vendus sont utilisés sur place pour la culture attelée, fréquente dans le nord du Bénin, car les superficies emblavées sont généralement plus importantes que celles du Sud.

En outre, les performances zootechniques enregistrées au sein des troupeaux bovins lors de la présente étude ont été globalement faibles. Le taux de mise bas n'a pas évolué avec le temps comparé à celui obtenu il y a vingt ans dans les troupeaux bovins traditionnels (Dehoux et Hounsou-Vè, 1993). Seul le taux de mortalité des veaux s'est amélioré avec le temps (tableau V) (Dehoux et HounsouVè, 1993) grâce à une meilleure couverture sanitaire et aux vaccinations systématiques des troupeaux bovins (deux fois par an, juin et décembre). Les taux de mise bas, de fécondité et la productivité numérique au sevrage enregistrés on été inférieurs à ceux obtenus chez les bovins Borgou de la ferme d'élevage de l'Okpara (tableau V ; Alkoiret et al., 2010a ; Gbangboché et Alkoiret, 2011). Ces écarts de performances avec la ferme de l'Okpara pourraient provenir des différences liées au suivi sanitaire des animaux, à l'alimentation, à la conduite du troupeau, ainsi qu'à la méthode de collecte de données (mémoire des éleveurs contre fiches de suivi).
Le taux de mise bas enregistré lors de cette étude a été similaire à ceux rapportés dans la littérature pour les troupeaux bovins des zones soudaniennes d'Afrique de l'Ouest. Dans une synthèse sur les systèmes de production bovine en Afrique subsaharienne, un taux de mise bas de $60 \%$ a été rapporté pour la région subhumide (moyenne de 48 travaux avec des taux variant entre 46 et $80 \%$; Otte et Chilonda, 2002). Des taux de mise bas de $54 \%$ ont également été rapportés chez les bovins du sud-est du Mali, caractérisé par un climat soudanien (Ba et al., 2011). En outre, dans des troupeaux majoritairement composés de races Azawak et Bororo en zone semi-aride du Niger les taux de mise bas varient entre 46 et $53 \%$ (Lesnoff et al., 2006). Le taux de mise bas de la présente étude, relativement faible par rapport au potentiel biologique (un veau/an/vache) reste sensiblement supérieur aux taux moyens de mise bas dans le Sahel (de l'ordre de $50 \%$ ).

Le taux de fécondité moyen relevé dans la présente étude a été similaire à celui des zébus (60\%) mais plus faible que ceux des races taurines N'Dama et Baoulé de Côte d'Ivoire (83 \% ; Sokouri et al., 2010). Le taux de mortalité des veaux a été similaire à celui des veaux dans les troupeaux de bovins du Sud-est du Mali (12\%; Ba et al., 2011) et des veaux N'Dama du sud du Sénégal (16 \% ; Ezanno et al., 2002). Cependant, un taux de mortalité des veaux beaucoup plus élevé a été enregistré (22\% ; Otte et Chilonda, 2002) pour les zones subhumides d'Afrique subsaharienne (moyenne issue de 44 travaux ; minimum $6 \%$, maximum $47 \%$ ).

Les taux de mise bas des races de zébus élevés principalement dans les régions sahéliennes et de leurs métisses ont été inférieurs à ceux des races taurines adaptées aux régions à forte infestation de glossines. Cette tendance est demeurée globalement la même dans le temps (Otte et Chilonda, 2002 ; Sokouri et al., 2010 ; tableau IV). Seuls les taux de mortalité ont globalement diminué avec le temps (Otte et Chilonda, 2002 ; Lesnoff et al., 2006 ; tableau IV) probablement en raison d'une meilleure couverture sanitaire. Malgré le faible

\section{Tableau IV}

Synthèse des performances de reproduction et de la structure des troupeaux bovins en Afrique de I'Ouest

\begin{tabular}{|c|c|c|c|c|c|}
\hline \multirow[t]{2}{*}{ Auteur } & \multirow[t]{2}{*}{ Pays } & \multirow{2}{*}{$\begin{array}{l}\text { Taux de mise bas } \\
(\%)\end{array}$} & \multirow{2}{*}{$\begin{array}{l}\text { Mortalité des veaux } \\
\text { de } 0 \text { à } 1 \text { an }(\%)\end{array}$} & \multicolumn{2}{|c|}{ Structure des troupeaux } \\
\hline & & & & Mâle & Femelle \\
\hline Nos résultats & Bénin & 64 & 10 & 25 & 75 \\
\hline Ba et al., 2011 & Mali & 49 & 10 & 40 & 60 \\
\hline Otte et Chilonda, 2002 & Zone subhumide & 60 & 22 & 36 & 64 \\
\hline Lesnoff et al., 2006 & Niger & $46-53$ & $10-16$ & 31 & 69 \\
\hline Ejlertsen et al., 2011 & Sénégal & 40 & $0-4$ & 30 & 70 \\
\hline Somda et al., 2005 & Gambie & - & - & 32 & 68 \\
\hline Akpa et al., 2012 & Nigeria & - & - & $25-40$ & $60-75$ \\
\hline Dehoux et Hounsouvè, 1993 & Bénin & 65 & 23 & 27 & 73 \\
\hline Alkoiret et al., 2010b & Bénin & 70 & 3 & 35 & 65 \\
\hline
\end{tabular}

Tableau V

Synthèse des performances de reproduction des bovins de race locale béninois

\begin{tabular}{|c|c|c|c|c|c|}
\hline & Milieu & $\begin{array}{c}\text { Mise bas } \\
(\%)\end{array}$ & $\begin{array}{c}\text { Fécondité } \\
(\%)\end{array}$ & $\begin{array}{c}\text { Productivité } \\
\text { numérique au sevrage } \\
(\%)\end{array}$ & $\begin{array}{l}\text { Mortalité des veaux } \\
\text { de } 0 \text { à } 1 \text { an } \\
(\%)\end{array}$ \\
\hline Nos résultats & Village & $64 \pm 15$ & $64 \pm 15$ & $57 \pm 18$ & $10 \pm 13$ \\
\hline Alkoiret et al., 2010 & Station & $70 \pm 15$ & $69 \pm 13$ & $67 \pm 13$ & $3 \pm 1$ \\
\hline Gbangboché et Alkoiret, 2011 & Station & 72 & 69 & 67 & - \\
\hline
\end{tabular}


taux de mise bas des bovins élevés en zones pastorales et semi-arides, la taille du cheptel bovin de ces régions qui représente $51 \%$ de l'effectif d'Afrique subsaharienne (Otte et Chilonda, 2002) permet d'approvisionner les marchés des pays côtiers, surtout par la transhumance transfrontalière. Les bovins de ces régions viennent ainsi renforcer la production locale des pays côtiers dont les races présentent un meilleur taux de mise bas mais avec un cheptel plus faible (environ $26 \%$ de l'effectif d'Afrique subsaharienne) (Otte et Chilonda, 2002).

Les performances d'élevage des troupeaux bovins traditionnels du nord du Bénin sont en général faibles et voisines de celles enregistrées dans les troupeaux de la sous-région. La structure des troupeaux est aussi similaire à celle enregistrée dans les troupeaux allaitants de l'Afrique au sud du Sahara. Cependant, pour les performances zootechniques, deux tendances se dégagent. Les troupeaux de Banikoara ont eu les meilleures performances, similaires à celles des races taurines adaptées aux régions à forte infestation par les glossines. Les performances des troupeaux des autres communes se sont rapprochées de celles des régions sahéliennes.

Les performances d'élevage sont en général faibles dans les troupeaux bovins béninois et ceux d'Afrique subsaharienne. Ces faibles performances sont généralement la conséquence d'un grand intervalle entre vêlages souvent causé par une alimentation déficitaire et des problèmes de santé. La gestion des effectifs des troupeaux ne permet pas à l'éleveur de bien entretenir les animaux ce qui a un impact négatif sur leurs performances et ne leur permet pas d'exprimer de façon optimale leur potentiel génétique.

\section{- CONCLUSION}

Les élevages de bovins situés au nord du Bénin présentent une structure identique à celle des troupeaux allaitants sahéliens. Cette structure caractérisée par la dominance des femelles permet de pérenniser le troupeau et de le gérer durablement. Les performances d'élevage sont relativement faibles, caractérisées par des taux de mortalité des veaux élevés et de faibles taux de mise bas et de fécondité. La structure de ces élevages, similaire à celle des pays sahéliens, permet néanmoins d'approvisionner les marchés du sud du pays, avant que les animaux issus des troupeaux sahéliens arrivent sur le marché des pays côtiers. Elle permet également aux agriculteurs de trouver sur place un outil de production (taurillons) pour la culture attelée, qui ménage la structure du sol, comparé à l'utilisation des tracteurs. Enfin, les performances zootechniques influencent la démographie des élevages bovins du nord du Bénin, permettant de répondre à la demande des pays côtiers, insatisfaite par l'offre locale.

\section{Remerciements}

Les auteurs tiennent à exprimer leur gratitude à la Coopération technique belge (CTB) pour le financement de cette recherche.

\section{REFERENCES}

Akpa G.N., Alphonsus C., Abdulkareem A., 2012. Evaluation of herd structure of white Fulani cattle holdings in Zaria-Nigeria. Afr. J. Anim. Biomed. Sci., 7 (1): 128-131

Alkoiret I.T., Awohouedji D.Y.G., Akossou A.Y.J., Bosma R.H., 2009 Typologie des systèmes d'élevage bovin de la commune de Gogounou au Nord-Est du Bénin. Ann. Sci. Agro. Bénin, 2 (12) : 77-98

Alkoiret I.T., Awohouedji D.Y.G., Gbangboche A.B., Bosma R.H., 2010a. Productivité des systèmes d'élevage bovin de la commune de Gogounou au nord-est du Bénin. Ann. Sci. Agro. Bénin, 14 (2) : 145-163

Alkoiret I.T., Awohouedji D.Y.G., Yacoubou A.M., 2010b. Paramètres démographiques des cheptels de bovins Borgou et N'Dama à la Ferme d'Elevage de I'Okpara au Nord-Est du Bénin. Int. J. Biol. Chem. Sci., 4 (5) : 1657-1666
Ba A., Lesnoff M., Poccard-Chapuis R., Moulin C.-H., 2011. Demographic dynamics and off-take of cattle herds in southern Mali. Trop. Anim. Health Prod., 43: 1101-1109

Daodu M.O., Babayemi O.J., Iyayi E.A., 2009. Herd composition and management practices of cattle production by pastoralists in Oyo area of Southwest Nigeria. Livest. Res. Rural Dev., 21 (5), 66

De Haan L., Biaou G., Fanou J., 1997. Le contexte national et régional. In : Agriculteurs et éleveurs au nord du Bénin. Ecologie et genres de vie (Ed. De Haan L.). Karthala, Paris, France, 175-195

Dehoux J.P., Hounsou-Vè G., 1993. Productivité de la race bovine Borgou selon les systèmes d'élevage traditionnels au Nord-Est du Benin. World Anim. Rev., 74/75 : 36-48

Djenontin J.A., 2010. Dynamique des stratégies et des pratiques d'utilisation des parcours naturels pour l'alimentation des troupeaux bovins au Nord-Est du Bénin. Thèse Doct., Université d'AbomeyCalavi, Faculté des sciences agronomiques, Bénin, 214 p.

Djenontin J.A., Amidou M., Baco N.M., 2002. Diagnostic sur la gestion du troupeau : gestion des ressources pastorales dans l'Alibori et le Borgou. In : Actes colloque Savanes africaines: des espaces en mutation, des acteurs face à de nouveaux défis (Eds Jamin J.Y., Seiny Boukar L., Floret C.), Garoua, Cameroun, 27-31 mai 2002. Cirad, Montpellier, France, 12 p.

Domingo A.M., 1976. Contribution à l'étude de la population bovine des états du golfe du Bénin. Thèse Doct., Ecole inter-Etats des sciences et médecine vétérinaire, Dakar, Sénégal, 148 p.

Ejlertsen M., Marshall K., Poole J., 2011. Gestion durable du bétail ruminant endémique $d^{\prime}$ importance mondiale en Afrique de l'Ouest : estimation des paramètres démographiques du cheptel au Sénégal. Rapport de recherche. ILRI, Nairobi, Kenya, 39 p.

Ezanno P., Ickowicz A., Faye B., 2002. Demographic parameters of $\mathrm{N}^{\prime}$ Dama cattle raised under extensive range management conditions in Southern Senegal. Rev. Elev. Med. Vet. Pays Trop., 55 (3) : 211-219

FAO, 2013. Country Stat, www.countrystat.org/home. aspx?c=ben\&ta=053SPD135\&tr=21, (consulté le 19/09/2013)

FAO, 2015. FAO Stat, faostat.fao.org/site/613/ DesktopDefault. aspx?PagelD=613\#ancor, (consulté le 03/02/2015)

Ferraton N., Touzard I., 2009. Comprendre I'agriculture familiale. Diagnostic des systèmes de production. Quæ, Versailles, France, 123 p.

Gbangboché A.B., Alkoiret T.I., 2011. Reproduction et production de lait des bovins de race Borgou et N’Dama au Bénin. J. Appl. Biosci., 46 : 3185-3194

IIED / SOS Sahel, 2010. Modern and mobile. The future of livestock production in Africa's drylands. IIED / SOS Sahel International, London, UK, pubs.iied.org/pdfs/12565IIED.pdf (consulté le 05/02/2013)

INSAE, 2003. Troisième recensement général de la population et de I'habitation. Synthèse des résultats. Ministère chargé du Plan, de la Prospective et du Développement, Cotonou, Bénin, www.insaebj.org/2012/doc/recensement/synthese_des_resultats_rgph3.pdf, (consulté le 12/11/12)

Lesnoff M., 2013. Survey methods to estimate demographic rates of tropical ruminant livestock herds. Review, limits and prospects [in French]. Rev. Elev. Med. Vet. Pays Trop., 66 (2): 57-67

Lesnoff M., Saley A., Adamou K., N’Djaffa H., 2006. Enquête démographique 2006 sur le cheptel domestique au Niger : sites du Fakara, de Gabi et de Zermou. Icrisat, Niamey, Niger / DGCD, Bruxelles, Belgique / ILRI, Addis Ababa, Ethiopia

Meyer C., éd. sc. 2013. Dictionnaire des sciences animales. Cirad, Montpellier, France, dico-sciences-animales.cirad.fr/ (consulté le 07/08/2013)

Otte M.J., Chilonda P., 2002. Cattle and small ruminant production systems in sub-Saharan Africa : A systematic review. Livestock Information Sector Analysis and Policy Branch. FAO, Rome, Italy, 98 p.

Sokouri D.P., Yapi-Gnaore C.V., N'Guetta A.S.P., Loukou N.E., Kouao B.J., Toure G., Kouassi A., Sangare A., 2010. Performances de reproduction des races bovines locales de Côte d'Ivoire. J. Appl. Biosci., 36 : 23532359

Somda J., Kamuanga M., Tollens E., 2005. Characteristics and economic viability of milk production in the smallholder farming systems in The Gambia. Agric. Syst., 85: 42-58 


\section{Summary}

Chabi Toko R., Adégbidi A., Lebailly P. Demography and zootechnical performance of traditional cattle farming in Northern Benin

The study was conducted with 150 Fulani herders in the departments of Borgu and Alibori in Benin. It aimed to determine the zootechnical parameters of traditional cattle farms. The survey collected information on the characteristics of cattle herds. The results showed a high proportion of females $(76 \%)$, an average herd size (66 animals) and the prevalence of the Borgu breed (86\%). The herds also showed a low breeding performance, characterized by a high calf mortality rate $(10 \%)$, and low reproductive parameters $(64 \%$ calving and fertility rates). In a year, 0.57 calf was weaned on average by its dam. The low performance of cattle farms in coastal countries such as Benin is consistent with that of pastoral areas, which is complementary in terms of meat supply to West African markets.

Keywords: Borgu cattle, herd, reproductive performance, livestock production, Benin

\section{Resumen}

Chabi Toko R., Adégbidi A., Lebailly P. Demografía y rendimiento de las explotaciones ganaderas tradicionales en el norte de Benín

El estudio fue realizado con 150 ganaderos Fulani en los departamentos de Borgu y Alibori en Benín. Su objetivo fue determinar los parámetros de producción de las explotaciones ganaderas tradicionales. La encuesta recoge información sobre las características de los hatos de ganado. Los resultados muestran una alta proporción de hembras $(76 \%)$, el tamaño medio del rebaño (66 animales) y la prevalencia de la raza Borgu $(86 \%)$. Los hatos presentaron también un bajo rendimiento de cría, caracterizado por una alta tasa de mortalidad de terneros $(10 \%)$ y parámetros reproductivos bajos, con índice de partos y de fertilidad de $64 \%$. Durante el año, 0,57 ternero es el promedio destetado por madre. El bajo rendimiento de las explotaciones ganaderas de los países costeros como Benín es consistente con los de las zonas de pastoreo, complementarias en términos de abastecimiento de los mercados de carne de Africa Occidental.

Palabras clave : ganado bovino Borgu, hato, reproductividad, producción pecuaria, Benin 
